\title{
Educação Infantil em diferentes contextos da Região do Pampa: traçando algumas reflexões
}

\section{La Educación Infantil en diferentes contextos de la Región del Pampa:} trazando algunas reflexiones

Infantile Education in different contexts of the Region of the Pampa: drawing some reflections

\author{
Alezandra Lima Nery Messias ${ }^{1}$ \\ Adriana Duarte Leon ${ }^{2}$
}

\begin{abstract}
Resumo
O presente trabalho tem por finalidade refletir sobre os diferentes contextos educacionais traçando uma linha temporal dentro da história da educação infantil da cidade de Bagé/RS/Brasil. Com intuito de compreender o que os documentos oficiais preconizam como fundamental e como à educação infantil vem sendo tratada na prática, considera-se neste trabalho o discurso oficial que afirma a educação infantil como uma das primeiras fases da educação para cidadania na educação básica. Nos últimos anos diversas políticas públicas têm focado no crescimento e no desenvolvimento das instituições acolhedoras. Assim, é fundamental analisar o que vem sendo feito dentro de algumas instituições que priorizam o atendimento conforme as necessidades fisiológicas da criança; como também as que priorizam o seu atendimento considerando o desenvolvimento cognitivo e intelectual. A metodologia para esta investigação trata-se de uma pesquisa qualitativa de cunho historiográfico que utiliza os seguintes instrumentos de coleta de dados: questionário, visitas e entrevistas com agentes públicos, professores, funcionários famílias dos alunos atendidos pela rede municipal de Educação Infantil do município de Bagé/RS/Brasil, além de visitas ao acervo público. Através da análise dos documentos e com a coleta de dados é possível investigar questões relativas à história da Educação Infantil, gestão e políticas educacionais no município de Bagé. Tudo isso, a partir do contexto em que o município se encontra em relação aos avanços e melhorias nesse segmento, dando ênfase aos investimentos técnicos e financeiros que são feitos, bem como, à qualidade da formação continuada de seus professores.
\end{abstract}

Palavras-Chave: criança, contextos educacionais, políticas públicas.

\footnotetext{
1 Discente do Mestrado em Educação e Tecnologia no IFSUL/câmpus Pelotas, Ivonira Maria Lima Nery, Ismar Antonio Nery. Rio Grande do Sul/RS, Brasil. alezandramessias@gmail.com, Pedagogia Educação Infantil pela FURG (Universidade Federal de Rio Grande), Pós-Graduação em Ludo Pedagogia, Leitura e Literatura FACEL (Faculdade de Administração, Ciências, Educação e Letras) Trabalho apresentado III Encontro Humanístico Multidisciplinar e II Congresso Latino-Americano em Estudos Humanístico Multidisciplinares, Unipampa, Jaguarão/RS, Brasil, 2017.

2 Professora do IFSUL/Câmpus Pelotas, atuando no MPET/IFSUL, linha de pesquisa "Educação, Cultura e Trabalho". Doutora em Educação pela UFMG e Mestre em Educação pela UFPel. E-mail: adriana.adrileon@gmail.com Trabalho apresentado III Encontro Humanístico Multidisciplinar e II Congresso Latino-Americano em Estudos Humanístico Multidisciplinares, Unipampa, Jaguarão/RS, Brasil, 2017.
} 


\begin{abstract}
Resumen
El presente trabajo tiene por finalidad reflexionar sobre los diferentes contextos educativos trazando una línea temporal dentro de la historia de la educación infantil de la ciudad de Bagé/RS/Brasil. Además de comprender lo que preconiza los documentos oficiales como siendo fundamentales. Como también discutir como la educación viene siendo tratada a lo largo de los años, ya que, se considera como una de las principales fases indispensables para la ciudadanía en la educación básica. También como las políticas públicas contribuyen para el crecimiento y el desarrollo de las instituciones. Así poder entender el proyecto de algunas instituciones donde priorizan el atendimiento de los niños conforme sus necesidades fisiológicas; aun los que priorizan el atendimiento considerando el desenvolvimiento cognitivo e intelectual. La metodología utilizada se trata de una pesquisa cualitativa de cuño historiográfico utilizando algunos elementos siendo ellos: cuestionario, visitas e entrevistas con agentes públicos, profesores, funcionarios e familias dos alumnos atendidos en la red municipal de Educación Infantil e aún visitas a los acervos. A través del análisis de los documentos posibilitaron investigar cuestiones relativas a la historia de la Educación Infantil, gestión e políticas educacionales del municipio. A partir del contexto en que el municipio se encuentra con relación a los avanzos y mejorías en este segmento, dando énfasis a los investimentos técnicos y financieros que son hechos, así como la cualidad en la formación continuada de sus profesores.
\end{abstract}

Palabras claves: niños, contextos educacionales, políticas públicas.

\title{
Resume
}

The present paper has the purpose of reflecting on the different educational contexts tracing a temporal line within the history of preschool education in the city of Bagé/RS/Brazil. In order to understand what the official documents advocate as fundamental and how preschool education has treated in practice, it is considered in this paper the official discourse that affirms the education of children as one of the first stages of education for citizenship in basic education. In recent years, public policies have focused on the growth and development of welcoming institutions. Thus, it is fundamental to analyze what has been done within some institutions that prioritize the care according to the physiological needs of the child; as well as those that prioritize their care considering cognitive and intellectual development. The methodology for this research is a qualitative research of a historiographic nature that uses the following instruments of data collection: questionnaire, visits and interviews with public agents, teachers, employees and families of the students who attend the municipal network of Preschool Education in Bagé/RS/Brazil, as well as visits to the public collection. By analyzing the documents and collecting data, it is possible to investigate issues related to the history of Preschool Education, management and educational policies in the city of Bagé. All this, based on the context in which the city is in relation to the advances and improvements in this segment, emphasizing the technical and financial investments that are made, as well as the quality of the continuing education of its teachers.

Key-Words: Kid, Contexts Educational, Public Politics.

\section{Introdução}

A educação infantil tem sido motivo de uma série de debates, seminários e fóruns quase sempre com objetivo de garantir qualidade e melhoria nos espaços educacionais. Contudo atende de maneira diferenciada as necessidades físicas e cognitivas das crianças cuja faixa etária se encontra de 0 a 5 anos.

Sendo ela a primeira etapa da educação básica, abrange a creche e a pré-escola, e se consolida como um espaço fundamental na formação do ser humano. De acordo com a Lei das Diretrizes e Bases da Educação Nacional (Lei 9394/96) no Art. 29 nos define que "a Educação Infantil, primeira etapa da educação básica, tem como finalidade o desenvolvimento integral da criança de zero a cinco anos, em seus aspectos físicos, psicológico, intelectual e 
social, complementando a ação da família e da comunidade. Com o passar do tempo foram criadas outras leis que buscavam garantir os direitos e serviram para normatizar a LDB/1996 e cobrar das autoridades a responsabilidade sobre a educação das crianças. Embora, nem sempre tenha sido dessa maneira que funcionou a educação em nosso país, como bem indica Ariés (1981) apud Santana (2014), na Idade Média, não existia o sentimento infância como temos hoje. Conforme o autor "esse sentimento iniciou-se com a paparicação da criança e evoluiu com a ascensão da burguesia que começou a ver a necessidade de moralizá-la através da educação" (AIRES, 1981).

Atualmente algumas instituições que atendem crianças nessa faixa etária marcam sua diferença naquilo que se propõem como: priorizar o atendimento conforme as necessidades fisiológicas da criança; ou as que priorizam o atendimento considerando o desenvolvimento cognitivo e intelectual. Em virtude disso, um fator indispensável nesse processo se encontra na formação do professor que atua nesses espaços infantis, pois esta formação é considerada um passo importante para uma proposta que agregue cuidar e educar, como exemplo, o cognitivo e fisiológico que se entrelaçam no seu atendimento, consolidando o que a legislação atual estabelece no acolhimento integral à criança e suas necessidades.

Os objetivos estabelecidos neste trabalho são: Investigar a constituição histórica dos espaços que atendem crianças pequenas na Rede Municipal de Bagé; Identificar na Secretaria Municipal de Educação de Bagé a legislação municipal que rege o funcionamento da educação infantil e Reconhecer os avanços e os desafios da Educação Infantil dentro do município. A metodologia utilizada para este trabalho trata-se de uma pesquisa qualitativa de cunho historiográfico ${ }^{3}$ com o uso de alguns elementos norteadores sendo eles: questionário, visitas e entrevistas com agentes públicos, professores, funcionários e famílias dos alunos atendidos pela rede municipal de Educação Infantil do município de Bagé/RS. Através da análise dos documentos e visitas que possibilitem investigar questões relativas à história da Educação Infantil, gestão e políticas educacionais no município de Bagé. A partir do contexto em que o município se encontra em relação aos avanços e melhorias nesse segmento, dando ênfase aos investimentos técnicos e financeiros que são feitos, bem como, à qualidade da formação continuada de seus professores.

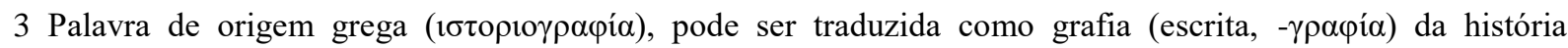

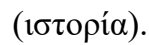




\section{Contexto da Pesquisa: Bagé a Rainha da Fronteira}

A cidade de Bagé está localizado no Estado do Rio Grande do Sul e é conhecida como a Rainha da Fronteira, pois se localiza na fronteira do Rio Grande do Sul. A origem do nome da cidade ainda é discutida, algumas vertentes dizem que o local onde hoje está situado o município viveu um cacique minuano chamado Ibajé, que teve seu corpo enterrado na região e teria dado o nome à cidade. Porém, a hipótese mais aceita até hoje é que o nome Bagé vem da linguagem indígena e que estaria relacionada com a ideia de "cerros", os índios tapes chamavam os Cerros de "bag." A cidade está localizada a $60 \mathrm{~km}$ do Uruguai, é o caminho mais curto entre Porto Alegre e Montevidéu, Bagé desempenhou um papel importante na história do Estado do Rio Grande do Sul por conta de sua posição geográfica. A seguir a figura um mostra a localização do município, suas divisas com outros municípios dentro do Rio Grande do Sul.

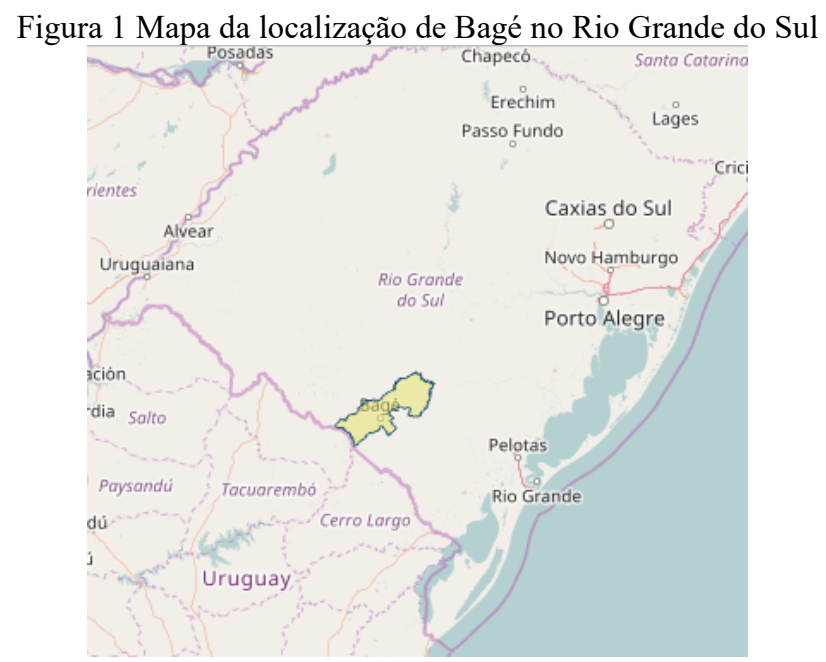

Fonte: https://mapasapp.com/mapa/rio-grande-do-sul/bage-rs

De acordo com informações extraídas pelo site da prefeitura Municipal de Bagé e do IBGE (Instituto Brasileiro de Geografia e Estatística) foram apontados os seguintes dados: em divisão territorial datada de 2001, o município é constituído de 5 distritos: Bagé, Joca Tavares, José Otavio, Palmas e Piraí. Também os dados oferecidos pelo último censo realizado no ano de 2010. A tabela a seguir mostra a população da cidade de Bagé e a sua densidade demográfica.

Tabela 2 Número de habitantes na cidade de Bagé

\begin{tabular}{|c|l|}
\hline População no último censo & Densidade demográfica \\
\hline 116,794 pessoas & $28,52 \mathrm{hab} / \mathrm{km}^{2}$ \\
\hline \multicolumn{2}{|c|}{ Fonte: $<$ https://cidades.ibge.gov.br $>$}
\end{tabular}


No gráfico abaixo demonstra o número de escolas de educação infantil no município de Bagé/RS desde a sua implantação nos anos oitenta até os dias atuais.

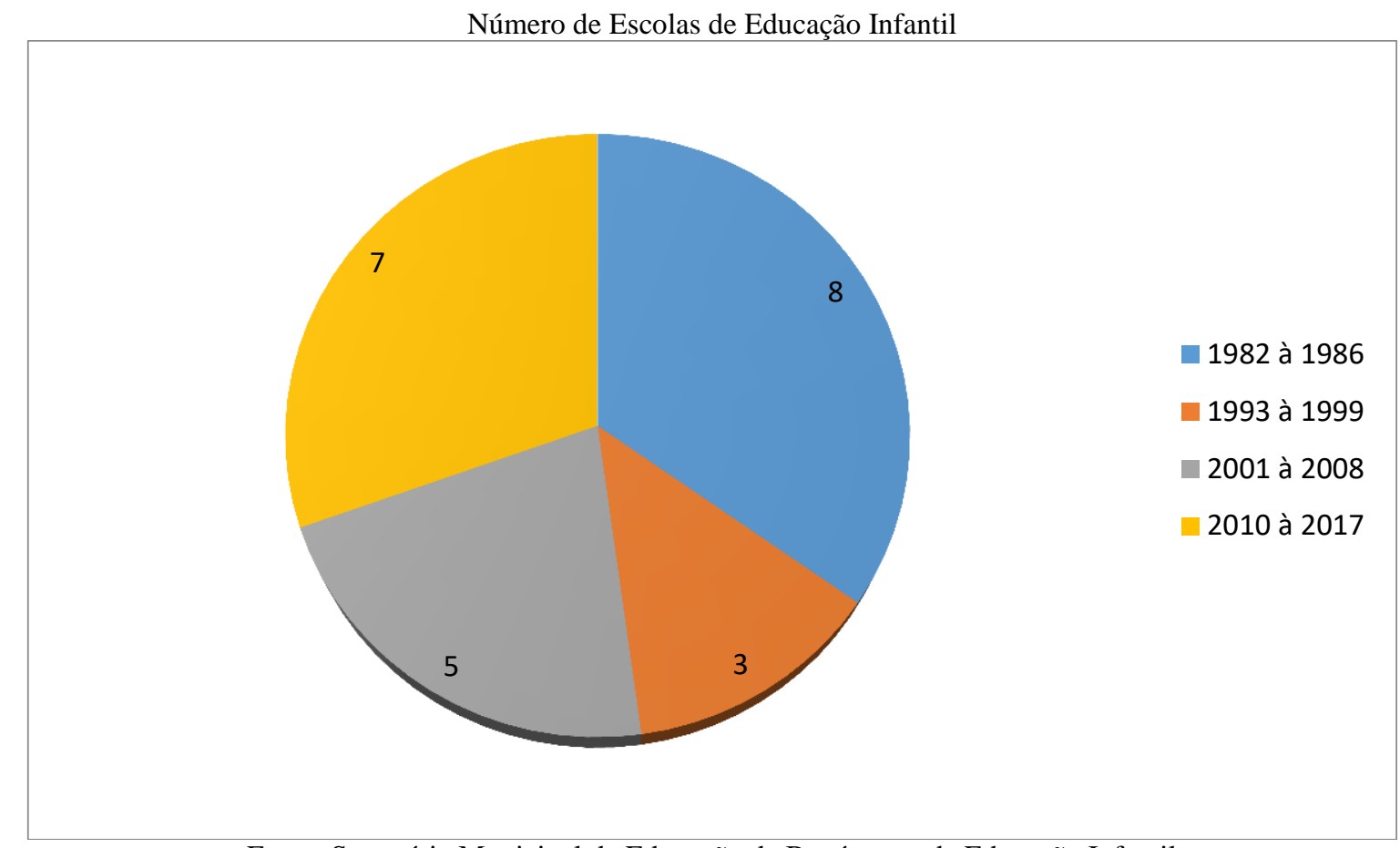

Fonte: Secretária Municipal de Educação de Bagé, setor de Educação Infantil

Ao analisar o gráfico observa-se que na década de oitenta foi um período de grande relevância para a educação infantil no município de Bagé/RS, onde oito espaços foram criados. Após a Constituição de 1988, como afirma Barbosa (2009), a Educação Infantil aparece com um caráter educativo ressaltado, foi garantido às crianças pequenas o direito à educação. Perceber-se também que a década de noventa reflete um período de certa estagnação em comparação as demais, pois, neste período apenas três espaços foram disponibilizados. Nas décadas seguintes os dados demonstram que houve aumento significativo, surgindo novos espaços de educação infantil no município.

Com tudo isso, acredita-se que um grande aliado para esse crescimento foi com a implementação das Diretrizes Curriculares Nacionais de Educação Infantil (DCNEI, 2009) de acordo com o documento à criança:

Sujeito histórico e de direitos que, nas interações, relações e práticas cotidianas que vivencia, constrói sua identidade pessoal e coletiva, brinca, imagina, fantasia, deseja, aprende, observa, experimenta, narra, questiona e constrói sentidos sobre a natureza e a sociedade, produzindo cultura. (BRASIL, 2009, p.12). 
Esses documentos corroboram com os direitos das crianças permitindo que a capacidade cognitiva, emocional, intelectual desenvolva-se de maneira lúdica e em sociedade criando espaços atrativos para esses sujeitos que formam parte de um futuro de uma nação.

\section{Conclusões}

Portanto, mesmo com todo avanço na área tecnológica, cientifica, política e econômica, pouco se consegue avançar na área da educação infantil. Ainda deixa-se, na maioria das vezes, em último plano não dando o seu devido valor, isso pode ser presenciado, em algumas escolas de educação infantil no município de Bagé onde uma parcela de professores, atua sem formação específicos para essa faixa etária, muitos vêem dos anos iniciais ou até mesmo de áreas afins como professor de português, artes e também ocorre falta de professor nas turmas sendo estas atendidas pelas atendentes ou berçaristas que em sua maioria não possuem formação na educação infantil. Com toda a legislação já criada ainda precisa-se caminhar mais para uma educação que valorize a educação infantil, respeitar essa etapa da vida permitindo que futuramente esse sujeito venha a ser um adulto com uma formação pedagógica de qualidade, despertando, assim, a vontade de avançar no conhecimento. Do ponto de vista das políticas públicas observa-se que houve alguns avanços na trajetória como parte fundamental da educação básica.

Considerando a minha vivência na área e primeiras entrevistas realizadas para está pesquisa é possível afirmar que a educação infantil no município de Bagé na rede pública municipal vem desenvolvendo algumas transformações, começando pelo termo creche que hoje não é mais usado para referir-se a educação infantil e sim escola, apresentando uma preocupação em oferecer um espaço de aprendizagem, de desenvolvimento integral da criança tanto no aspecto físico, psicológico, intelectual e social, um exemplo desse avanço para a educação infantil no município foi a criação da sala de atendimento especializado para atender alunos com algum tipo transtorno ou deficiência, outro fator positivo nesta na formação dos professores oferecida pela secretaria de educação durante o ano letivo de 2017.

A comunidade escolar busca ou pelo menos tenta buscar melhorias em todas as áreas da educação sendo ela na infraestrutura, na qualificação dos professores e funcionários. Através dos recursos oferecidos pelos governos municipais e federais. Isso se torna possível através das pesquisas e estudos que vão se formando dentro das instituições de ensino. 


\section{Referências}

ARIÈS, Philippe. História social da criança e da família. Traduzido por Dora. Flaksman. Rio de Janeiro: LTC, 1981.

BASTOS. M. H. C. Jardim de Crianças - o pioneirismo do Dr. Menezes Vieira (1875-1887). IN: MONARCHA. C. Educação da infância brasileira 1875 - 1983. Campinas: Autores Associados, 2001.

BOGDAN, R.; BIKLEN, S. Investigação qualitativa em educação: uma introdução à teoria e aos métodos. Porto: Portugal: Porto Editora, 1994.

BRASIL. Lei de Diretrizes e Bases da Educação Nacional. Lei n 9.394, de 20 de dezembro de 1996.

CAMPOS. M. M. ROSEMBERG. F. FERREIRA. I.M. Creches e pré-escolas no Brasil. São Paulo: Cortez, 1993.

BRASIL. Ministério da Educação. Secretaria de Educação Básica Parâmetros nacionais de qualidade para a educação infantil/Ministério da Educação. Secretaria de Educação Básica - Brasília. DF v.l; il. 1. Educação Infantil. 2. Ensino Fundamental. 1. Título. 2006.

SÁ, Elizabeth Figueiredo. As representações da Infância Brasileira e a Escolarização da Infância. In: SÁ, Elizabeth Figueiredo. De criança a aluno: as representações da escolarização da infância em Mato Grosso (1910 -1927). Cuiabá: EDUFMT, 2007. (pg. 27 57)

SANTANA, Djanira Ribeiro. Infância e educação: a histórica construção do direito das crianças. Revista HISTEDBR On-line, [S.l.], v. 14, n. 60, p. 230-245, abr. 2015. ISSN 16762584. Disponível em:

<https://periodicos.sbu.unicamp.br/ojs/index.php/histedbr/article/view/8640557/8116>. Acesso em: 04 ago. 2017. doi:http://dx.doi.org/10.20396/rho.v14i60.8640557.

KULHMANN JR. M. Infância e educação infantil: uma abordagem histórica. Porto Alegre: Mediações, 2001.

VIVEIROS, Kilza Fernanda Moreira de. A criança negra no Maranhão: uma leitura a partir da infância afrodescendente no Brasil. In: CONGRESSO BRASILEIRO DE HISTÓRIA DA EDUCAÇÃO, 6., 2006, Goiânia. Anais Goiânia: Sociedade Brasileira de História da Educação, 2006. Disponível em :

$<$ https://periodicos.ufrn.br/educacaoemquestao/article/viewFile/4467/3658> acesso em 21/08/2107. 